\title{
A investigação sobre manuais escolares e materiais curriculares
}

\author{
Jesús Rodríguez Rodríguez \& Denébola Álvarez \\ Seoane
}

Resumo

Este trabalho apresenta uma classificação das diferentes perspetivas e linhas de pesquisa que foram analisadas e desenvolvidas nos últimos anos sobre materiais didáticos. Essas perspetivas foram as seguintes: (i) papel e influência dos manuais escolares e dos materiais curriculares nas práticas de aula; (ii) discurso ideológico subjacente aos materiais; (iii) análise dos aspetos formais nos manuuais escolares e noutros materiais curriculares; (iv) impacto das políticas e processos de reforma educacional sobre as características desses materiais; (v) função e estudo desses materiais nas diferentes áreas do currículo e nas didáticas específicas; (vi) análise do manual escolar e de outros materiais como discurso curricular e de profissionalização docente; (vii) processos de comercialização dos manuais escolares e programas de gratuidade; (viii) diretrizes para avaliação e análise de manuais escolares; e (ix) investigação sobre a elaboração de materiais pedagógicos alternativos. Neste estudo, apresentamos algumas investigações que nos parecem interessantes vir a desenvolver sobre manuais escolaires nos próximos anos.

Palavras-chave:

manuais escolares; materiais didácticos; tecnología educativa. 


\section{Research on textbooks and learning materials}

Abstract: This paper presents a classification of the different perspectives and research lines analyzed and developed in recent years regarding textbooks. The perspectives considered were: (i) The role and influence in the classroom practices of textbooks and curriculum materials; (ii) Underlying ideological speech in educational materials; (iii) Analysis of the formal aspects in textbooks and other instructional materials; (iv) The impact of policies and educational reform processes on the characteristics of materials; $(v)$ The function and the study of materials in different areas of the curriculum and specific didactics; (vi) Analysis of textbook and other curricular materials speech and teacher professionalism; (vii) Processes marketing of textbooks and free programs; (viii) Guidelines for evaluation and analysis of texts; (ix) Research on the development of alternative materials. The paper concludes by mentioning some research that seems interesting to develop in relation to textbooks in the coming years.

Key-words: textbooks; didactical materials; educational technology.

\section{La recherche sur des manuels scolaires et le matériel pédagogique}

Resumé: Cet article présente une classification des différentes perspectives et axes de recherche analysés et développés au cours des dernières années concernant les manuels scolaires. Les perspectives considérés ont été les suivantes: (i) Le rôle et l'influence des manuels et de matériels pédagogiques dans les pratiques de classe; (ii) Discours idéologique soustendu dans le matériel pédagogique; (iii) L'analyse des aspects formels dans les manuels scolaires et autres matériels didactiques; (iv) L'impact des politiques et des processus de réforme de l'éducation sur les caractéristiques des matériaux; (v) La fonction et l'étude des matériaux dans différents domaines du programme scolaire et didactique spécifiques; (vi) Manuel d'analyse et d'autres matières scolaires telles que la parole et le professionnalisme des enseignants; (vii) Processus de commercialisation des manuels scolaires et les programmes libres; (viii) Lignes directrices pour l'évaluation et l'analyse des textes; (ix) La recherche sur le développement de matériaux alternatifs. Le document conclut en mentionnant quelques recherches qui semblent intéressantes à développer par rapport aux manuels scolaires dans les années à venir.

Mots-clés: manuels scolaires; materiaux didactiques; tecnologie educative.

\section{La investigación sobre libros de texto y materiales curriculares}

Resumen: Este artículo presenta una clasificación de las distintas perspectivas y líneas de investigación que se analizaron y desarrollaron en los últimos años en relación con los libros de texto. Las líneas de investigación consideradas fueron las siguientes: (i) El papel y la influencia en las prácticas de aula de los libros de texto y materiales curriculares; (ii) Discurso ideológico subyacente en los materiales educativos; (iii) Análisis de los aspectos formales en los libros de texto y otros materiales curriculares; (iv) La repercusión de las políticas y los procesos de Reforma Educativa sobre las características de los materiales; (v) La función y el estudio de los materiales en las diferentes áreas del curriculum y en las didácticas específicas; (vi) Análisis del libro de texto y otros materiales como discurso curricular y de profesionalidad docente; (vii) Procesos de comercialización de los libros de texto y programas de gratuidad; (viii) Guías de evaluación y análisis de textos; e (ix) Investigaciones sobre la elaboración de materiales alternativos. El artículo concluye mencionando algunas líneas de investigación que parece interesante desarrollar en relación con los libros de texto en los próximos años.

Palabras clave: libros de texto; materiais didácticos; tecnoloxía educativa. 


\section{Introdução}

Para realizar este trabalho, procedemos à leitura e análise de várias revisões bibliográficas que se realizaron sobre esta temática. Os referentes fundamentais de partida foram as análises de revistas sobre manuais escolares e materiais existentes em Espanha e Portugal, as bases de dados ERIC, DIALOG, ISOC e a base de dados da Faculdade de Harward, entre outras. Também se reviram, dum modo especial, as publicações presentes no e-Journal da International Association for Research on Textbooks and Educational Media (IARTEM) (http://biriwa.com/iartem/ejournal/) e as publicações derivadas dos congressos da IARTEM realizados en Caen, Tønsberg, Santiago de Compostela, Kaunas, Ostrava (consultáveis em http://iartemblog.wordpress.com/publications/) e Berlím (próxima publicação www.iartem.org), assim como as publicações dos seus congressos regionais sobre "A comprensão e a melhoria da função dos livros de texto e dos meios educativos em âmbitos com escassos recursos para o meio" que teve lugar em Antananarivo, "Construção dos conflitos e da paz em/por manuais e meios educativos" realizado em Montreal e "Os desafíos para superar a desigualdade social: o papel dos livros de texto e os meios educativos" que se realizou en Curitiba (https://iartemblog.files.wordpress.com/2014/01/iartem_volume_curitiba_2012.pdf). Recentemente, acaba de ser lançado o livro de Atas do Congresso Regional da IARTEM de Colombia (www.iartem.org). Outro colóquio de referência foi o que teve lugar en Montreal organizado pela Université du Québec em torno da questão Le manuel scolaire: D'ici et d'ailleurs, d'hier à demain.

Igualmente, tivemos presentes algumas investigações do contexto sulamericano, das quais se destacam as desenvolvidas na Colômbia e nalguns países da América Central, como Costa Rica e Honduras, por ser aqui que a UNESCO desenvolve um maior número de iniciativas dirigidas ao fomento da leitura e da elaboração de materiais curriculares impressos. Convém salientar os dois seminários internacionais sobre textos escolares organizados no Chile pelo Ministério da Educação, em que se apresentaram um número considerável de relatórios e comunicações relacionados com a temática deste encontro, assim como os encontros recentes realizados en Lisboa pela Universidade Lusófona: $5^{\circ}$ Coloquio Internacional Manuais Escolares - Manuais Escolares, E-Manuais e Investigação em Didática e $6^{\circ}$ Coloquio Intenacional Manuais Escolares - Recursos educativos digitais, gestão educacional e aprendizagem. Além disso, incluímos na nossa valoração outros trabalhos e relatórios, que consideramos de especial relevância e significado com relação à investigação sobre os manuais escolares e materiais curriculares, assim como os estudos e as publicações realizados polo Georg-Eckert-Institut, que podem consultar-se em http://www.gei.de/en/publications/. 
Importa assinalar as seguintes limitações em relação com a revisão a que se alude neste artigo: dificuldade em analisar teses de mestrado e de doutoramento, pois em muitos países e universidades não existem bases de dados adequados que permitam aceder a este tipo de informação e dificuldade em aceder às bases de dados dalguns países. No caso dalguns países, não foi possível encontrar bases de dados que facilitassem o acesso aos diferentes estudos e, noutros casos, não existem publicações que tenhan como objetivo fundamental a revisão da literatura existente sobre o tema.

\section{Linhas de investigação}

Vejamos, a seguir, uma síntese das principais linhas de investigação analisadas.

\subsection{O papel e a influência dos manuais escolares e dos materiais curriculares nas práticas de aula}

Entre as preocupações fundamentais que se assinalan neste grupo de investigações, há que salientar o uso e o inventário dos materiais didáticos, o propósito de querer conhecer até que ponto o ensino se encontra condicionado polos manuais escolares e pelos materiais curriculares e o uso que se faz destes recursos na aula $e$ nos centros educativos. Em linhas gerais, os resultados da investigação refletem uma clara dependência dos manuais por parte do professorado. Quanto às razões desta dependência, mencionam-se fatores de experiência, a quantidade de tempo destinado à instrução e as perceções dos professores sobre a validade dos manuais escolares. Alguns estudos também revelam que muitos professores usam e seguem literalmente os manuais do professor que acompanham os manuais escolares destinados aos alunos. Outros põem de relevo a dependência dos professores de determinado tipo de materiais curriculares impressos.

Alguns resultados da investigação relativizam o impacto e o protagonismo dos manuais escolares no currículo. Por exemplo, Area \& Correa (1992), a respeito da opinião dos professores sobre o uso dos manuais escolares nas escolas, comentan que os resultados refletem que uma boa parte do professorado não admite que um modelo de uso exclusivo e tradicional dos manuais escolares seja representativo da sua prática. Quanto às práticas de uso dos manuais escolares, Henson (1981, citado por Area, 1994) identifica diversas práticas de uso do manual mais ou menos estandardizadas que este autor classifica como utilizar o manual escolar como currículo, combinação do manual com outros materiais, substituição do manual escolar por outros meios ou materiais. Outra série de práticas que poderiam encontrar-se seriam: dependência do manual escolar, omissão seletiva centrada no fundamental, gestão por objetivos, etc.

Quanto ao modo de uso de professor que se encontrou con maior frequência, destaca-se o uso do manual escolar como currículo e como autoridade máxima na aula. 
Em definitivo, poderíamos dizer que os professores usam os manuais escolares como principal recurso de instrução; não obstante, não se pode afirmar que este é o único meio empregado. Em linhas gerais, utiliza-se o manual escolar como currículo e em combinação con outros materiais e os professores manifestan uma maior dependência dos materiais comerciais que doutros materiais.

\subsubsection{Causas e motivos que determinan a seleção que os professores fazem de uns e outros materiais.}

Um problema específico de investigação relacionado con esta linha é a seleção de materiais didáticos. A classificação de Woodward, Elliot \& Nagel (1988) proporcionanos uma síntese interessante dos aspectos abordados nesta linha de investigação, entre os quais se destacam os seguintes: a influência que teve o mercado na venda dos manuais escolares, a escolha do processo e as técnicas ou estratégias de avaliação que se usaram para selecionar os manuais escolares.

Por outro lado, a maioría dos estudos põem em relevo o escasso papel que se concede aos grupos de especialistas académicos nos processos de seleção dos materiais e o papel determinante que desempenham os grupos de especialistas e administradores do setor editorial. Igualmente, convém salientar os diferentes modos de considerar os processos de seleção nos diferentes países, nalguns casos o papel das administrações resulta determinante e noutros não se deteta nenhum interesse em tentar melhorar os processos de seleção.

\subsection{Discurso ideológico subjacente aos materiais educativos}

Trata-se de investigações em que se analisa o conteúdo implícito nos materiais didáticos: questões relacionadas com os valores, com o conteúdo moral e cívico, com a discriminação sexual e ideológica, etc. Entre os aspetos estudados nos materiais impressos, este é um dos mais habituais, ainda que manifestamente em diferentes revisões existam uma série de campos ainda por explorar, tais como o influxo dos editores na elaboração dos manuais. A maioria dos trabalhos referenciados por Woodward, Elliot \& Nagel (1988) são categorizados por Watt dentro dos três seguintes enfoques: como os autores influíram na socialização política, como as ideologías se refletem nos manuais escolares usados nas ciências sociais e como os conteúdos dos materiais de leitura básicos transmitem valores culturais. Vejamos em continuação uma síntese de algumas das principais modalidades de estudo que configuram esta linha de investigação.

\subsubsection{Estereótipos sobre a mulher reproduzidos nos materiais didáticos}

A maioria dos estudos relacionados con esta temática põe em relevo o tratamento discriminatório que sofre a mulher nos materiais curriculares, e refletem a necessidade 
duma revisão dos recursos (veja-se, por exemplo, Knudsen, 2010 e Sanchez Bello, 2010). Em linhas gerais, nestes estudos assina-se que o sexismo e a discriminação se manifestam claramente nestes materiais. Nas análises das autoras mostra-se como tácita, subliminal, consciente ou inconscientemente, a mulher aparece em inferioridade e claramente discriminada, tanto no aspecto qualitativo como no quantitativo: faltam representações de mulheres e das suas contribuições em cada área de conhecimento, o âmbito político está protagonizado pelos homes, utilíza-se o masculino como genérico na maioria das ocasiões, há uma falta de adequação da figura da mulher à situação atual, o feminino não tem uma definição autonómica e faz-se sempre en função do masculino e a presença das mulheres nos exemplos e nas definições dos dicionários é realmente escassa.

\subsubsection{Discriminação étnica ou racial}

Dentro desta subepígrafe incluem-se aquelas investigações que fazem referência à análise de como aparecem tratados aqueles grupos que tradicionalmente se considerarom minoritários e sobre os quais parece que recaiu uma certa marginalidade, pela escassa atenção que se Ihes presta socialmente e pelo tratamento destes coletivos nos materiais educativos (grupos de imigrantes, populações indígenas, etc.). Trabalhos como o de Senegacnik (2012) são uma amostra deste tipo de estudos, em que ficam a claro as discriminações que sofrem alguns coletivos sociais nos recursos educativos.

\subsubsection{Cultura da sustentabilidade}

Resultam de especial interesse os trabalhos do Grupo Ecologistas em Ação (Herrera López, 2007), que estudam a presença de estereótipos e valores antiecológicos numa amostra de manuais escolares de ensino básico e secundário. Nos seus trabaIhos põe-se em relevo, entre outros assuntos, o futuro ocultado pela abrangência da tecnología e do desenvolvimento, a irrelevância das soluções propostas e a ocultação das questões relevantes. Caurín \& Martínez (2013) analisaram o conceito de biodiversidade numa amostra de manuais escolares de segundo ciclo de educação básica. $\mathrm{Na}$ perspetiva da criação de propostas para a educação ambiental, tem especial relevância o projeto Climántica, que pode consultar-se en http://www.climantica.org/, e foi divulgado também em congressos e em revistas científicas e profissionais.

\subsection{Análise dos aspetos formais nos manuais escolares e noutros materiais curriculares}

Trata-se de um campo de investigação que goza duma sólida tradição investigadora. Neste sentido, alguns trabalhos centram-se, por exemplo, en analisar as cores e os efeitos empregados nos materiais instrucionais, em examinar a ideología dos livros didáticos, em observar como a forma e o significado dos materiais textuais afetam o 
currículo en termos de linguagem, etc. Em linhas gerais, estas investigações põem em relevo a necessidade de que os materiais devem prestar maior atenção às ilustrações e de que devem evitar imagens contraditórias, racistas e descontextualizadas. Nalguns destes trabalhos (Väisänen, 2008), faz-se referência a como o simbólico dos materiais se constitui em poderosa ferramenta através da qual se plasmam determinadas ideologías e valores.

\subsection{O impacto das políticas e processos de reforma educacional sobre as características dos materiais}

As reformas educativas propostas nos sistemas educativos supõem, de um modo ou de outro, mudanças nas decisões sobre os materiais (elaboração, possível reconcetualização destes, etc.). Woodward, Elliot \& Nagel (1988) citam vários estudos de investigação sobre os efeitos que os materiais instrucionais produzem durante os períodos de reforma educativa. Da análise destas investigações, os autores deduziram questões tais como o interesse do governo em subvencionar contribuições de novos manuais escolares e em querer adaptá-los à filosofía das reformas, e também se observa um certo interesse por parte da administração em querer estabelecer medidas de controle e de rendibilidade sobre os materiais que se estão a produzir.

Nos últimos anos, desenvolveram-se algumas investigações com o propósito de analisar os materiais desenvolvidos nos contextos das reformas educativas. Entre os resultados destas investigações, torna-se manifesto o seguinte: as empresas que editaram material com motivo de colocação em marcha de reformas do sistema educativo não optaram, em geral, por inovações substanciais; o manual escolar continua a ser concebido, em grande parte, como manual único; o conceito de ciclo não foi realmente assumido; os aspetos mais bem resolvidos são os formais e os piores são os da atenção à diversidade; em muitos materiais, há grandes défices no âmbito das intenções educativas, ainda que nalguns materiais isso seja um aspeto especialmente cuidado, e noutros aparecem certos estereótipos (especialmente nas ilustrações) que se podem considerar discriminatórios; a maioria de editoriais mostram uma linha orientadora e formulações unificadas nos distintos materiais em que se repercute boa parte dos materiais curriculares analisados mas que devem considerar-se inadequadas.

\subsection{A função e o estudo dos materiais nas diferentes áreas do currículo e nas didáticas específicas}

Trata-se de investigações que se centraram en analisar como aparece concetualizada ou representada cada uma das áreas do currículo nos materiais curriculares de física e química, geografía, ciências naturais, matemáticas, lingua e literatura, música... É dizer, como aparece, por exemplo, o conceito de medida, magnitude, gramática ou 
estrutura da mensagem. Em geral, detetam-se importantes erros concetuais, muitas vezes fruto da falta de trabalho interdisciplinar.

Em linhas gerais, podemos dizer que em cada área há claros défices quanto aos conteúdos que deverían incluir. Na maior parte dos trabalhos nota-se a necessidade de que se desenvolvam mais trabalhos específicos em cada área sobre os materiais curriculares. Também existe uma crescente investigação centrada no desenvolvimento de competências (digital, socioemocional, cidadã, aprender a aprender, matemática, comunicação linguística, cultural e artística, entre outras).

\subsection{Análise do manual escolar e doutros materiais como discurso curricular e de profissionalização docente}

Martínez Bonafé \& Rodríguez Rodríguez (2010) assinalam que este é um campo de especial interesse, mas escassamente investigado. Os trabalhos desenvolvidos nesta linha põem em relevo a desprofissionalização docente para que contribuem os manuais escolares. E encontra-se também aquilo que os docentes consideran as decisões do manual escolar como algo que não se deixa submeter a crítica, aparecendo nestas propostas a segurança e garantia de um bom que-fazer profissional.

\subsubsection{Economia política do material curricular: investigações sobre editores e materiais didáticos}

Dentro deste grupo de investigações, poderíamos incluir aquelas que têm que ver com o modo de como "influem os intereses pessoais, institucionais e tradicionais sobre o processo de desenvolvimento do manual escolar" (Johnsen, 1996, p.226). Um dos autores que mais investiu neste âmbito é Apple, que analisa, entre outros aspetos, as relações de poder que têm lugar no mundo editorial, as caraterísticas dos autores que participam na elaboração dos manuais escolares, a distribuição do trabalho nas editoras em função do sexo, entre outros muitos aspetos. Em Espanha, prestou especial atenção a esta questão Martínez Bonafé (2002 e 2004) e Moyano (2010) realizou uma breve síntese do sector editorial. No contexto portugués, pode consultarse o estudo de Sousa \& Lourdes (2010).

\subsubsection{Legislação sobre a aprovação ou a legalização dos manuais escolares e a prática educativa}

Neste âmbito convém destacar un conjunto de trabalhos relacionados com a aprovação ou com a legalização dos manuais escolares e as repercussões que isto supõe no contexto educativo. Da totalidade de estudos de que Johnsen (1996) faz revisão neste campo, cabe salientar as seguintes questões: existem políticas de aprovação bastante díspares quanto ao modo como se controla e supervisa o material educativo 
e a existência de material variado e diverso faz com que os sistemas e os critérios de aprovação devam sofrer mudanças importantes.

No contexto espanhol, é destacável o trabalho de Gimeno (1995) realizado a partir da análise dos ISBN e de observação da evolução da edição de manuais escolares no contexto espanhol. O autor esmiuça e põe em relevo os interesses comerciais que se encontram detrás do mercado dos manuais escolares, as diferenças que existem entre o mercado dos materiais escolares e os materiais universitários e também as relações que se estabelecem entre essa cultura mercantilista e as disposições administrativas existentes. Verdugo (1998) analisa a evolução experimentada pela indústria editorial na Galiza e destaca que os manuais escolares ocupam o principal segmento de produção em língua galega.

No contexto latinoamericano, importa destacar uma forte linha de investigação sobre o Programa Nacional do Livro Didático (http://www.nppd.ufpr.br/nppd/). No contexto europeu, encontramos estudos sobre a situação do manual escolar em países como Eslováquia, Grécia ou Suécia. Igualmente, também encontramos estudos relevantes na Coreia, Kénia ou Estados Unidos (vejam-se alguns exemplos na revista Ejournal, biriwa.com/iartem/ejournal/). A investigação de Watt (2007) constitui-se num referente fundamental sobre a evolução da indústria editorial no contexto estadounidense.

\subsection{Processos de comercialização dos manuais escolares e programas de gratuidade}

A maioria dos estudos que se aproximam a este âmbito fazem-no do ponto de vista do interesse do mercado. Martínez Bonafé \& Rodríguez Rodríguez (2010, pp.253-254) assinalam que "falta uma maior profundidade das implicaçõess que supõe a adoção de umas ou outras medidas do ponto de vista do desenvolvimento curricular e, especialmente, as licitações às propostas de inovação e renovação pedagógica que questionam a hegemonía do manual escolar". Contudo, os estudos e os relatórios existentes contribuem para analisar os modelos e as propostas de gratuidade existentes e as implicações económicas para o setor editorial e para as famílias.

Alguns exemplos dos relatórios realizados em Espanha são os da Associação Nacional de Editores de Livros e Material de Ensino (http://www.federacioneditores. org e http://www.anele.org); os relatórios e os estudos da Confederação Espanhola de Organizações de Donas de Casa, Consumidores e Usuários, especialmente o denominado "O que custa um filho" (http://www.ceaccu.org/publicaciones/) ou o estudo realizado no ponto de vista da institução parlamentar por Bayona Aznar (2009). A Revista Mexicana de Investigação Educativa dedicou um número aos manuais escolares gratuitos e tratou temas como a das ambiguidades destas políticas, a promoção da equidade e a igualdade de oportunidades, a evolução e a atualidade do programa de gratuitidade ou o tratamento da história e dos povos indígenas nos manuais escolares 
gratuitos (consultar Consejo Mexicano de Investigación Educativa, 2011 em http:// www.comie.org.mx/v1/revista/portal.php com contribuições de Anzures; Barriga; Grediaga; Pacheco; Navarro \& Cayeros; ou Vargas).

\subsection{Diretrizes para avaliação e análise de manuais escolares}

Neste ponto, poderíamos considerar trabalhos que têm que ver com a elaboração de propostas e modelos de avaliação e, ao mesmo tempo, de estudos realizados para constatar o efeito ou a utilização dessas propostas de avaliação. Neste sentido, encontramos uma diversidade de achegas sobre guias e modelos de avaliação (veja-se, por exemplo, Djurovic, 2009; Reints \& Wilkens, 2010). O principal problema está na falta de formação para o seu uso, o desconhecimento da sua existência e, em muitos casos, não interesa a difusão deste tipo de instrumentos que podem ser um perigo para os interesses dalguns produtores de materiais ao verem seriamente criticados os seus recursos educativos.

\subsection{Investigações sobre elaboração de materiais alternativos}

Esta linha de investigação recolhe experiências e práticas de criação de recursos educativos alternativos ou complementares aos manuais escolares, em muitos casos com o propósito de adaptar o currículo aos alunos ou de fazer presente o local no conteúdo curricular. Nesta linha, e vinculada também ao campo dos recursos educativos em contextos não escolares, está a desenvolver-se na Universidade de Santiago de Compostela uma investigação que tem como propósito a catalogação e a análise dos materiais didáticos publicados por administrações locais, cujos primeiros achados indicam que os materiais didáticos de produção municipal não apoiam o trabalho nos centros escolares e instituções educativas, onde continua a predominar o manual escolar, e é possivelmente um dos principais problemas aquela escassa difusão.

Também é frequente que neste tipo de investigações se analisem fundamentalmente as caraterísticas dos materiais e recursos didáticos que foram elaborados polos próprios professores como práticas alternativas aos manuais escolares, e é pena que estas propostas dos docentes sejam minoritárias e "não disponham de crédito administrativo nem de produção e difusão adequadas e requeiram ainda, em muitos casos, formação específica dos professores, obstáculos que dificultan em grande medida a sua propagação e disseminação" (Travé \& Pozuelos, 2008, p.6).

\subsection{Livro de texto eletrónico}

Em relação con este tema, merece especial consulta sobre esta linha de investigação a obra de Rodríguez, Bruillard \& Horsley (2015) em que se mostra um número notável de trabalhos. A revista Currículum y Formación del Profesorado (Vol. 20, n 1 , 2016), publicou a monografia Entre libros de texto impresos y libros de texto digitales: 
debates y desafíos (coordenado pelos profesores Doutores: Jesús Rodríguez Rodríguez da Universidade de Santiago, Jaume Martínez Bonafé da Universidade de Valencia e Tânia Braga García da Universidade Federal do Paraná-Brasil). Além disso, é de grande interesse neste campo a monografia da revista Educatio Siglo XXI coordenado por José Peirats Chacón e Graciela A. Esnaola Horacek com o título Digitalización de los contenidos curriculares, (http://revistas.um.es/educatio/issue/view/14181/showToc). E também devemos mencionar a revisión realizada por Rodríguez Rodríguez e Martínez Bonafé em Libros de Texto y Control del Curriculum en el contexto de la sociedad digital $(2016,36)$.

Em geral, as aproximações que se fizeram ao livro de texto digital revelam a inclusão nestes recursos de exercícios e atividades interactivas e a incorporação de elementos atrativos e assinalam o potencial de futuro. Porém, um número considerável de investigações mostram que uma boa parte desses livros que aparecem com a denominação de livros de texto digitais realmente são edições de manuais escolares impressos mas recolhidos em formato PDF, sem que ofereçam maiores alternativas didáticas que os manuais escolares convencionais e, em geral, têm a dificuldade de não permitir fazer anotações.

Junto a esta crítica ao livro de texto digital, vêm outras duas (Martínez Bonafé \& Adell, 2003): uma primeira vinculada ao custo económico da adaptação do processo editorial e uma segunda relacionada com a potencionalidade de uso real pela diversidade de contextos e relacionados ainda com a fenda digital. Sobre a primeira, uma das grandes dificuldades que parecem sinalizar-se como motivo para que non pareçam detetar-se inovações substanciais nalguns dos libros de texto digitais analisados está relacionada com as dificuldades de tipo económico, pelo alto custo que supõe incorporar novos profissionais aos processos de produção do livro. Sobre a segunda, os problemas de conexão são uma dificuldade real para o uso potencial dos livros de texto digitais. Nalguns países resulta realmente complicado pensar no emprego do livro de texto digital ao não dispor de eletricidade que permita o emprego de computadores ou doutros dispositivos eletrónicos que possam facilitar o emprego destes materiais.

\subsection{Outras investigações}

Nos últimos anos, encontrámos algumas investigações centradas na inclusão das Tecnologias da Informação e Comunicação como alternativa ao manual escolar, na comparação das implicações nos processos de apropriação dos conteúdos dos materiais em suporte impresso e digital e no emprego de recursos eletrónicos na aula. Outras linhas de investigação que se seguiram nos últimos anos estiveron relacionadas com a análise da identidade nos manuais escolares, com o estudo dos recursos educativos utilizados nas regiões fronteiriças, com a valorização dos materiais didáticos empregados para o estudo das línguas estrangeiras, com as crenças e atitudes dos 
professores sobre os recursos e com a formação dos professores face a esses recursos, entre outras.

\section{Considerações finais}

As investigações sobre o uso, a função e as características dos manuais escolares e dos materiais curriculares deixam manifesto que existem importantes carências destes recursos. De igual modo, convém salientar a alta dependência dos professores do manual escolar, o que supõe que os professores tenham por hábito deixar as decisões sobre os materiais curriculares nas mãos das editoras. Para lá da análise dos enfoques e das investigações realizadas, parece recomendável fomentar a investigação em âmbitos como: análise dos processos e das estratégias editoriais, processos de distribuição, caraterísticas e análise dos materiais produzidos no contexto educativo não formal, impacto das novas tecnologías nos processos de planeamento, uso e avaliação dos materiais curriculares impressos e dos materiais curriculares digitais, análise comparativa dos materiais elaborados nas distintas comunidades autónomas, caraterísticas dos materiais nas zonas fronteiriças, estudo das decisões que se tomam nos centros educativos no âmbito institucional sobre o tema e a formação de assessores, inspetores e outros profissionais de apoio aos professores sobre os materiais curriculares, etc. E também, tal e como apontam Martínez Bonafé \& Rodríguez Rodríguez (2010), a escassez de estudos que triangulem prática docente, uso do manual escolar e qualificación profissional, assim como estudos centrados nos processos editoriais. Por outro lado, assinalam algumas linhas como ausentes:

tampouco se prestou especial atenção à diversidade política e cultural relacionada com o estado das autonomias, nem se conhecem investigaçõess especialmente preocupadas pela perceção de que os alunos têm dos seus própios livros. Por outra parte, resultaria interessante conhecer o impacto dos recursos que se vão incorporando nas escolas na dinâmica organizativa. [...] Também seriam precisos estudos comparados entre comunidades autónomas; estudos sobre as caraterísticas e as análises dos materiais produzidos em contextos educativos não escolares; sobre o impacto das novas tecnologías nos processos de planeamento, uso e avaliação dos recursos curriculares impressos; ou a análise da formação de assessores, inspectores e outros profissionais de apoio aos professores sobre os materiais curriculares, ou o modo com esta questão se aplica nos currículos da formação inicial do professores, entre outros (Martínez Bonafé \& Rodríguez Rodríguez, 2010, p.259). 


\section{Referências Bibliográficas}

Area, M. (1994). Los medios y materiales impresos en el currículo. In J. M. Sancho (Coord). Para una tecnología educativa (pp. 85-113). Barcelona: Horsori.

Area, M. \& Correa, A. D. (1992). La investigación sobre el conocimiento y actitudes del profesorado hacia los medios. Una aproximación al uso de los medios en la planificación y desarrollo de la enseñanza. Qurriculum, 4, 79-100.

Bayona Aznar, B. (2009). Reflexiones y propuestas sobre las políticas de gratuidad de los libros de texto en España. Revista de las Cortes Generales, 76, 39-113.

Caurín, C. \& Martínez, M. J. (2013). Análisis del concepto de biodiversidad en los libros de texto de segundo ciclo de primaria en la Comunidad Valenciana (España). Perfiles Educativos, 141 (35), $97-$ 114.

Djurovic, A. (2009). Criteria for Evaluation and selection of Textbooks in Serbia. In M. Horsley \& J. McCall (Ed). Peace, Democratization and Reconciliation in Textbooks and Educacional Media. Ninth International Conference on Textbooks and Educational Media (pp. 221-229). Tønsberg: IARTEM.

Gimeno, J. (1995). Materiales y textos: Contradicciones de la Democracia Cultural. In J. G. Mínguez \& M. Beas (Coord). Libro de texto y construcción de materiales curriculares (pp. 75-130). Granada: Proyecto Sur.

Herrera López, Y. (2007). El curriculum oculto antiecológico de los libros de texto. Ambienta: la revista del Ministerio de Medio Ambiente, 69, 22-40.

Johnsen, E. B. (1996). Libros de texto en el calidoscopio. Estudio crítico de la literatura y la investigación sobre los textos escolares. Barcelona: Ediciones Pomares.

Knudsen, V. S. (2010). With gender awareness: Improving the reading of gender in educational texts and ilustrations. IARTEM e-Journal, 3 (1), 1-17.

Martínez Bonafé, J. (2002). Políticas del libro de texto escolar. Madrid: Morata.

Martínez Bonafé, J. (2004). Profesorado y reformas en el umbral del siglo XXI. Madrid: Miño y Dávila.

Martínez Bonafé, J. \& Adell, J. (2003). Viejas pedagogías, nuevas tecnologías. Cuadernos de Pedagogía, 326, 99-105.

Martínez Bonafé, J. \& Rodríguez Rodríguez, J. (2010). El curriculum y el libro de texto. Una dialéctica siempre abierta. In J. Gimeno Sacristán (Coord). Saberes e incertidumbres sobre el curriculum (pp. 246-268). Madrid: Morata.

Moyano (2010). El sector del libro de texto en España: análisis de la situación presente y perspectivas de futuro. In J. Rodríguez Rodríguez, M. Horsley \& S. V. Knudsen (Ed). Local, National and Transnational Identities in Textbooks and Educational Media. Tenth International Conference on Textbooks and Educational Media (pp. 47-49). Santiago de Compostela: IARTEM.

Peirats Chacón, J. \& Esnaola Horacek, G.A. (Coord) (2015). Digitalización de los contenidos curriculares. Educatio Siglo XXI, 33 (3). 
Reints, A. J. C. \& Wilkens, H. J. (2010). Evaluating the quality of textbooks from the perspective of the learning process. In J. Rodríguez Rodríguez, M. Horsley \& S. V. Knudsen (Ed). Local, National and Transnational Identities in Textbooks and Educational Media. Tenth International Conference on Textbooks and Educational Media (pp. 467-474). Santiago de Compostela: IARTEM.

Rodríguez, J., Bruillard, E. \& Horsley M. (Eds) (2015). Digital Textbooks, What's New? Santiago de Compostela: IARTEM / Servizo de Publicacións USC. http://www.usc.es/libros/index.php/spic/ catalog/book/759

Rodríguez Rodríguez, J. \& Martínez Bonafé, J. (2016). Libros de Texto y Control del Curriculum en el contexto de la sociedad digital. Cad. Cedes, 36 (100), 319-336.

Rodríguez Rodríguez, J., Martínez Bonafé, J. \& Braga García, T. (Coord.) (2016). Entre libros de texto impresos y libros de texto digitales: debates y desafíos. Profesorado. Revista del Currículum y Formación del Profesorado, 20 (1), 35-41.

Sánchez Bello, A. (2010). Gender Stereotypes in textbooks. In J. Rodríguez Rodríguez; M. Horsley \& S. V. Knudsen (Ed). Local, National and Transnational Identities in Textbooks and Educational Media. Tenth International Conference on Textbooks and Educational Media (pp. 356-361). Santiago de Compostela: IARTEM.

Senegacnik, J. (2012). European traditional national minorities in Slovenian geography textbooks compared with textbooks from other European countries. IARTEM e-Journal, 5 (1), 22-32.

Sousa, J. \& Lourdes, M. (2010). Between the market and the school: Textbook approval, selection and evaluation in Portugal. In J. Rodríguez, M. Horsley \& S. V. Knudsen (Ed). Local, National and Transnational Identities in Textbooks and Educational Media. Tenth International Conference on Textbooks and Educational Media (pp. 490-495). Santiago de Compostela: IARTEM

Travé, G. \& Pozuelos, F. J. (2008). Consideraciones didácticas acerca de las líneas de investigación en materiales curriculares. A modo de presentación. Investigación en la escuela, 65, 3-10.

Väisänen, J. (2008). Visual text in Finnish history textbooks. In E. Bruillard, B. Aamotsbakken, S. V. Knudsen \& M. Horsley (Ed). Caught in the web or lost in the textbook. Eight International Conference on Learning and Educational Media (pp. 297-304). Caen: IARTEM.

Verdugo, R. M. (1998). A indústria editorial em Galiza. Santiago de Compostela: Laiovento.

Watt, M. (2007). Research on the Textbook Publishing Industry in the United States of America. IARTEM e-Journal, 1 (1), 1-16.

Woodward, A., Elliot, D. L. \& Nagel, K. C. (1988). Textbook in school and society: an annotated bibliography and guide to research. New York: Garland Publishing. 
Jesús Rodríguez Rodríguez

Professor Titular de Universidade (Departamento de Pedagoxía e Didáctica). Sua pesquisa está enfocada no desenho e avaliação de materiais didáticos. Ele é um membro do grupo de pesquisa Stellae (USC) (http://stellae.usc.es),

Diretor da Revista Galega de Educación (http://www.nova-escola-galega. org) e Presidente da IARTEM (www.iartem.org).

Email: jesus.rodriguez.rodriguez@usc.es

\section{Denébola Álvarez Seoane}

Professora, pedagoga e mestrada em processos de formaçao com especializaçao em inovaçao e qualidade com experiência de trabalho comunitário e em escolas. Atualmente, faz uma tese de doutorado sobre a avaliação de material didático desenvolvido com o apoio das administrações locais. Faz parte do grupo de pesquisa Stellae (http://stellae.usc.es).

Email:carmendenebola@gmail.com

Correspondência

Jesús Rodríguez Rodríguez

Rúa Pro. Vicente Fráiz Andón, s/n. Campus Vida. 15782 Santiago de Compostela. A Coruña, España.

Data de submissão: Setembro 2016

Data de avaliação: Dezembro 2016

Data de publicação:Julho 2017 
